\title{
Research on Generalization of Patch in Water System of Land Use
}

\author{
TangZhe \\ China University of Geosciences \\ Faculty of Information Engineering \\ Wuhan, China, 13476155645 \\ tangzhe0727@sina.com
}

\begin{abstract}
In this paper we discussed the problems about water elements generalization in Cartographic Generalization, It includes some methods about the selection and simplification in the water elements generalization, extracting the axis-line of river, and the generalization of land use patch. At last, we design and implement the generalization of land use patch, include the generalization of little patch and generalization in water system. And receive a good effect.
\end{abstract}

Keywords- GIS; Cartographic generalization; Features in water system; Patch

\section{INTRODUCTION}

Cartographic generalization is an important and difficult problem in the field of GIS. In layman's terms, Cartographic generalization is a process that simplify the information of map with the change of the scale, and keep the spatial information clear and succinct at the same time.

The research scope of the map generalization is very wide. As one of the basic elements of the map, the river system generalization plays a very important role in the map generalization, mainly including river selection, river simplification and river integration, etc. The river system generalization has had many research results, for example, the models for determining river selection indexes including univariate regression model, multivariate regression model and root extraction model, etc ${ }^{[5]}$. the simplification algorithm of the linear river system including vertical interval algorithm, Douglas-Peucker algorithm ${ }^{[3]}$, Li-Openshaw ${ }^{[4]}$ algorithm and other algorithms; the method of changing facet river to linear river including D-triangle skeleton line method for extracting the central axis of the double line stream ${ }^{[6]}$.

The article mainly introduces the algorithm of selection and simplification of the facet river system and the linear river system, In addition, the article emphatically analyzes the problem of the river system data generalization of the land use, include the little patch generalization and the river system road generalization, and carries out the secondary development and achievement by the MapGIS software, and obtained better result.

\section{THE GENERALIZATION OF LINEAR RIVER SYSTEM}

The rivers of the river system have various shapes, so it is complicated to determine the rivers to be selected. The basic principle of river selection: the main stream is preferential; the selected river can reflect the structure features of the river system; and the selected river can keep density difference of river network ${ }^{[7]}$.

In general, the researcher can take the river length as the selection standard and keep the rivers with larger length. For example, provided that a certain length has been taken as the length standard, the rivers whose length is larger than the above value will be selected, while others will be removed from the research scope. However, the river length can not be regarded as the only one basis for river selection, for length of some rivers is smaller than the length standard, but they play an important role in the map or have special significance. On the contrary, some rivers larger than the length standard can be removed from the research scope. Thus we shall comprehensively take the following elements including river network density, river network types, river network length and so on, as the standard for river selection.

There are many kinds of generalization and simplification algorithms of the line elements which have their own merits and demerits. Such as vertical interval algorithm, DouglasPeucker algorithm ${ }^{[3]}$, Li-Openshaw ${ }^{[4]}$ algorithm. But after the research and summarization of the experts, the D-Palgorithm is regarded as one of the most classic algorithms of the line element generalization and simplification. The algorithm can not only simplify mass of data, but also can have a fast running speed. But it can only be used for the condition of small variation range of the plotting scale. So if the plotting scale has a large variation range, the better part of feature points selected by this algorithm are inconformity to that selected by the manual generalization method. So it is difficult to describe the geophysics features. But we can make some improvements, such as, calculate the maximum distance point of the polygon curve and divide the curve; determine the distance threshold value based on the calculation method of $\mathrm{Li}$-Openshaw algorithm for the diameter of the smallest visible object $(\mathrm{SVO})^{[8]}$; in case that there are several points with same offset, the curve should be divided into several sections based on the 
multi-section division method, rather than "one divides into two".

\section{THE GENERALIZATION OF FACET RIVER SYSTEM}

Generally, all the facet rivers will be kept for research and different threshold value standard will be selected and used for different plotting scale. As for the water reservoirs, lakes, ponds and other facet water areas, they can be selected or abandoned according to certain standards, rather than integration. Certainly the shapes of facet river system are various, so we shall operate on a case-by-case basis. For example, as for the river classification ${ }^{[9]}$ referred in the relevant documents and materials, the researcher shall take a comprehensive consideration of the grade information and length of the rivers to prevent the river system area in significance being deleted.

The facet polygon simplification plays an important role in the map generalization. Since the facet polygon has the features including diversified shapes and complex organization structure, etc. and the automation of the cartographic generalization has not been achieved yet, the polygon summarizing simplification is the key point of the automatic cartographic generalization and spatial analysis field. Recently, researchers and experts increasingly attach importance to polygon data processing based on the Delaunay triangulation network subdivision model which becomes a popular research subject $^{[10]}$.

As for the facet river system simplification, the extraction of the facet river system skeleton line is a relatively complicated process. One extraction algorithm is the facet river system skeleton line extraction algorithm on the basis of the Delaunay triangulation network. The basic idea of this algorithm: firstly, establish the Delaunay triangulation network of the double line stream; secondly, structure the skeleton line of Delaunay triangulation network based on the above step; finally, replace the central axis of the double line stream with the skeleton line.

\section{THE GENERALIZATION OF LAND USE PATCH}

The land use data is the typical element of the polygon type map. It has the following features including full coverage, no overlap and no seam and has the type layering classification in semantically(Bader \& Weibel,1997). As for the generalization, the simplification of spatial geometrical characteristics and integration results of the type layer in semantically should be achieved in advance. While the type reduction and geometrical shape simplification are completed, the researcher should keep the data features of full coverage and no overlap and no change of the relative percentage of each type of land use area in statistically. It is difficult to satisfy all the above conditions. The simplification of the polygon area one after the other results in the simplification of the area shape, but the common boundary between the areas will be inconsistent after the simplification and seam or overlap may come out. In case the small areas whose area is smaller than the threshold value but which may play an importance role in the system are directly deleted and replaced by the adjacent land area, it may lead to the greatly decrease of the percentage of this land type, which will damage the relative balance of the original land type.

Now we will discuss the little patch generalization and the river system road generalization.

\section{A. the little patch generalization}

The main role of the little patch generalization is to delete the patch in the map whose area is smaller than the area threshold value. But after the patch is deleted, the position where the patch is located will become empty and the completeness of the map will be damaged. So the process of the little patch generalization is not only to delete the eligible patch, but also to merge the deleted area into the surround area so as to keep the completeness and seamless of the map.

Basic procedures of the little patch generalization: firstly, the researcher should select the eligible little patches based on the area threshold value, and then merge the little patch into the surround patches: the researcher should merge the same type of patches (if any) into the little patch; if not, the researcher should merge the similar patches into the little patch; if the above two assumptions can not be satisfied, the researcher should merge the little patch into the patch with the maximum common edge of it. After the integration of the patches, the researcher should generally classify the merged little patches into three classes: 1 . as for the patch whose area is smaller than the threshold value, the researcher should merge it into its surrounding area; 2 . as for the patch whose area is smaller than the threshold value but locates in the polygon boundary, the researcher should merge it into its adjacent area or the area with the maximum common edge; 3 . as for the patch whose area is larger than the threshold value, the researcher should directly cut off it and add it into the layer.

The flow chart shows the design process of generalization (the whole process is completely automatic):

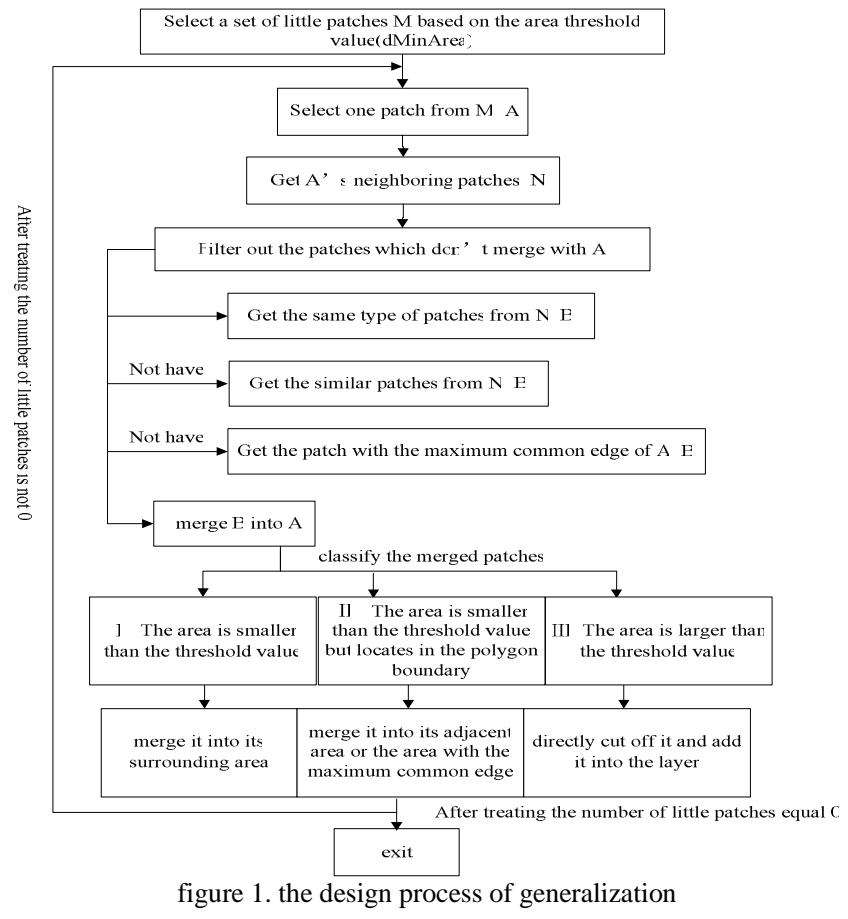


Through this method of processing, not only removes small area of patch, achieved the simplifying of information, but also maintain the integrity of map.

The following illustration shows the result of experiment: Through the little patch generalization we can get the figure 3 from the original figure 2, we can see the patch in figure 2 have been deleted or merged, and the completeness of the map not be damaged.

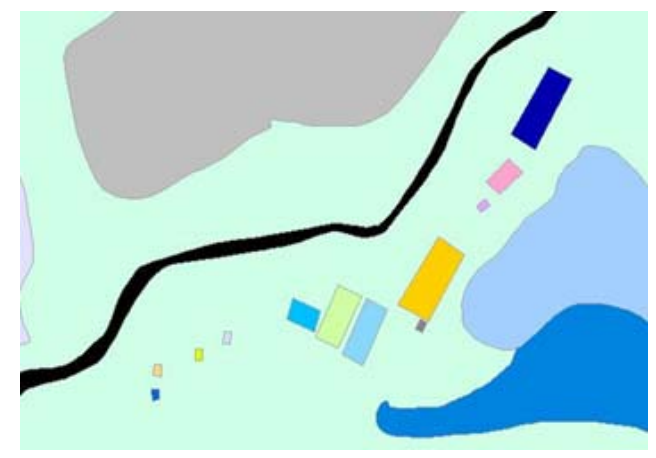

Figure2. Before the little patch generalization

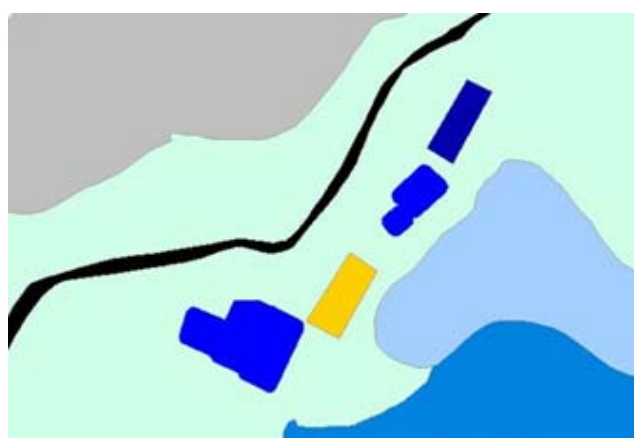

Figure3. After the little patch generalization

\section{$B$. the river system road generalization}

The main contents of the land use patch generalization also include the generalization of river system road. The main task of the river system road generalization is to keep the river system whose width is larger than the threshold value in its original state and simplify the river whose width is smaller than the threshold value, i.e. simplify the facet river system into linear river system. The same to the previous described little patch generalization, if the researcher simply change the facet river system into linear river system, the completeness of the map will be damaged and there will be bland area in the map. In this case, the surrounding area of the river system road should be taken into consideration and the researcher should fill the bland area after the simplification of the facet river system simplification.

Main procedures of river system road generalization: firstly, the researcher should achieve the adjacent area of the river system area and the curve section of the river system area and get each section point by traversing the curve sections; and then extract the central axis and traverse section point group; make the extended line of the relative curve sections and make the line to intersect with the central axis; and then remove the curve section in the river system area. In this case, the river system is simplified into the central axis of the river system. In order to keep the completeness of the map, the research should keep the original adjacent area into the interim feature class and then add the central axis and extended line into it. The new feature layer can be got after the researcher cuts off all the curve sections of this feature class and rebuilds them.

Since the complexity of the river system area selection, too much considering factors for the river system simplification and the complexity of river system road data, the generalization process is relatively complex. So we generally use the interactive generalization and the main procedures are listed as follows(Taking the second development of MapGIS K9 for example):

Procedures of generalization:

1) Achieve the adjacent area of the river system area R, then authored a collection of area(include the river system area $\mathrm{R}): \operatorname{Reg}\{\mathrm{A} / \mathrm{B} / \mathrm{C} \ldots \mathrm{R}\}$.

2) Get the set of curve sections $\operatorname{Arc}\{\operatorname{Arc} 1 / \operatorname{Arc} 2 \ldots\}$ by looking up the curve section of the river system area R.

3) Get each section point by traversing the curve sections, and get a set of point Pnt 1\{\} after deleting redundant points, then judge the number of curve sections which connect to each section point in Pnt1\{\}, add the section point to the connection Pnt2 \{\} if the number is larger than two, and relate to its appropriate curve section by map container. (figure 4)

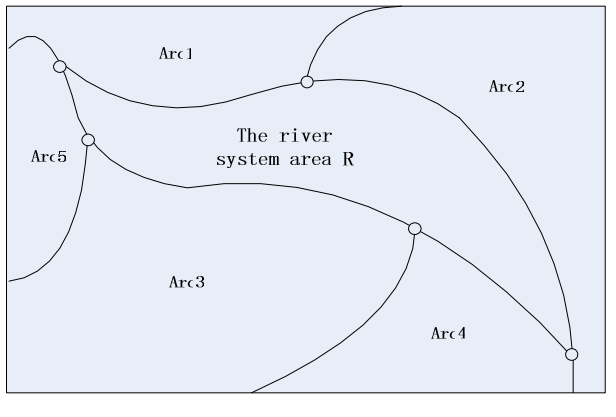

Figure 4

4) Extract the central axis of river system area R, and add to the layer.

5) Traverse Pnt2\{\}, make the extended line of the relative curve sections and make the line to intersect with the central axis. Get the set of intersections $\mathrm{T}\{\}$ and the set of extended line $\operatorname{LinT}\{\}$.(figure 5)

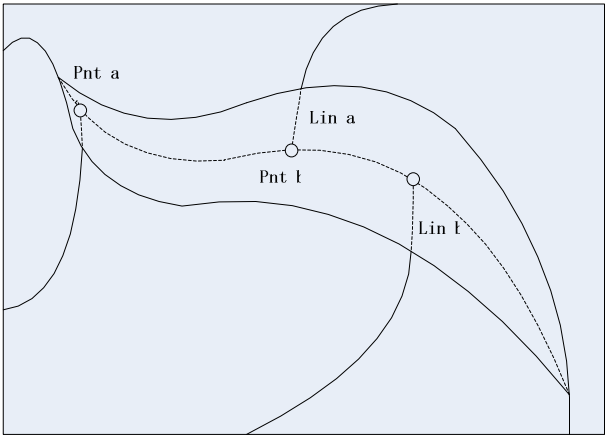

Figure 5 
6) Add Reg \{\} to an interim layer feature class AClass and extract Label point in AClass, put these points into Pnt\{PntA/ PntB/PntC/... PntR \}.

7) Delete the set of curve section Arc $\{\operatorname{Arc} 1 / \operatorname{Arc} 2 \ldots\}$, and add the central axis and extended line LinT \{\} into AClass.

8) A new feature layer BClass can be got after you cut off all the curve sections of AClass and then rebuild them.(figure 6)

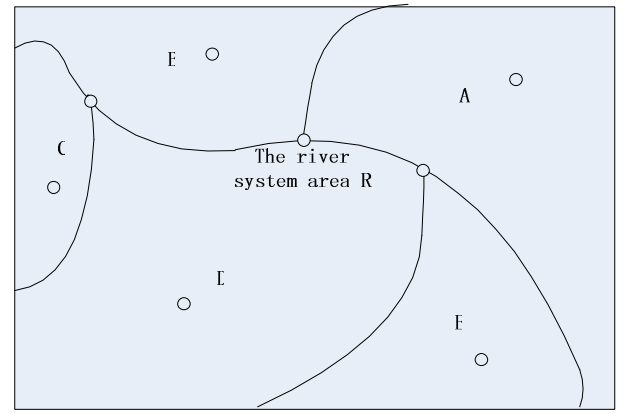

Figure 6

After merging the river system area good effects have been achieved, the example picture is as follows.

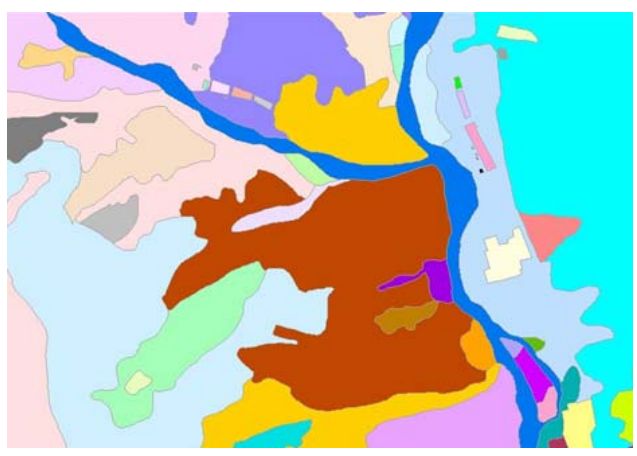

Figure7. Before merging the river system area

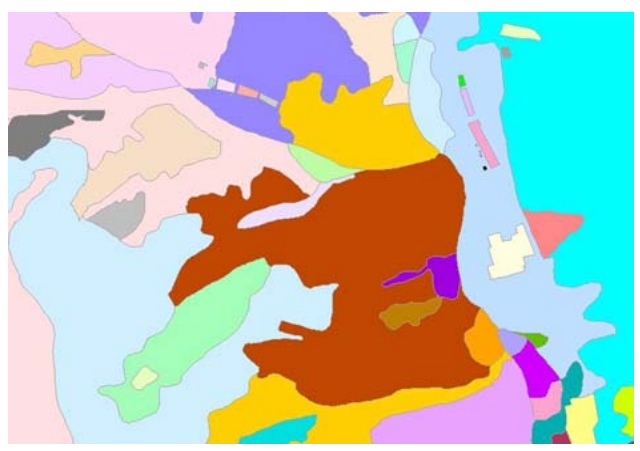

Figure8. After merging the river system area
After experiment, The central axis of river system area $\mathrm{R}$ have been successfully extracted, and the river system area have been merged into its surrounding area.

\section{CONCLUSION}

The cartographic generalization is a tough subject in the GIS field. It is related to the development level of the national GIS technology and cartography, so it has a very important practical significance for the research of the cartographic generalization. The article mainly introduces the river system element generalization of the cartographic generalization and simply describes the selection and simplification of the facet river system and the linear river system. In addition, the article emphatically analyzes the river system data generalization of the land use and carries out the secondary development and achievement by the MapGIS software. And relatively good effects have been achieved for special data. But the completeness and robustness of the function shall be strengthened in order to make it appropriate for more common conditions. It requires the researcher to carry out further study and perfection.

\section{REFERENCES}

[1] Ying-shen, Guo Ren-zhong, Yan Hao-wen, Lin Heng-gui. Framework Design and Implementation of Model-Oriented Cartograpkic Generalization[ J ] . Acta Geodaetica et Cartographica Sinica, 2002 , 31 (4) : 3442349.

[2] Dettori G, Puppo E-Designing a Library to Support Mod el-Oriented Generalization [ A ] In : Proceedings 5th ACM Workshop on Advances in GIS [C] , November 1516, Washington, DC(USA), 1998 : 34 -39.

[3] Douglas D1H1 \& Peucker T1K1 Algorithms for the Reduction of Points Required to Represent a Digitized Line or Its Carricature[ J] 1 Canadian Cartographer, 1993, ( 10): 112-1221.

[4] Li ,Z.-L, and Openshow, S. 1992. Algorithms for Automated Line Generalization Based on a Natural Principle of Objective Generalization . INT. J. Geographical Information Systems. 6 (5) : 373 389 .

[5] Zhang Qing-nian, Generalization of Drainage Network with Density Differences [A], Acta Geodaetica et Cartographica Sinica 2006.(5)

[6] AI Zi-xing, WU He-hai , AI Ting-hua , et al . The Application of Delaunay Triangulation in River Net Automatic Generalization [J] . Geo2Information Science , 2003 , (2) : 39-42.

[7] HE Zong-yi. Principles and Methods of Cartographical Models for Data Processing [M] . Wuhan : Wuhan University Press , 2004.

[8] Lei Wei-gang. Liu Da-jie, Tong Xiao-hua, Discussion about uncertainty of spatial line feature generalization algorithms, Engineering of Surveying and Mapping, 2004.(6) 7 10.

[9] Zhang-Gang, Li-Dong, Research on Automatic Cartographic Generalization in Water System [A] Water Conservancy Science and Technology and Economy. 2009. (4) 302 304.

[10] Ai Ting-hua, Guo Ren-zhong, Chen Xiao-dong, Simplification and Aggregation of Polygon Object Supported by Delaunay Triangulation Structure [J] Journal of Image and Graphics. 2001. (7) 703 710 